\title{
OSTEODISTROFIA HEREDITÁRIA DE ALBRIGHT: UM RELATO DE CASO
}

\author{
Bruna Batista Mesquita de Carvalho' \\ Isadora Benevides Silva Gondim Nascimento I \\ Cláudia Barros Gonçalves Cunha" \\ * Julya de Assis Sousa Morais ${ }^{\prime}$ \\ Leonardo Meira de Carvalho'
}

\section{RESUMO}

O pseudohipoparatireoidismo ocorre devido a uma resistência periférica à ação do paratormônio, cursando com hipocalcemia. Quase todos os casos são do tipo IA, correspondente a Síndrome de Osteodistrofia Hereditária de Albright. O presente estudo tem como objetivo relatar o caso de um paciente de 31 anos que apresentava calcificações nos rins, sistema nervoso central e articulações, além de dosagem baixa do paratormônio, compatível com a Síndrome supracitada. Trata-se de um estudo retrospectivo, observacional e descritivo com abordagem quantitativa, de um paciente portador de Osteodistrofia Hereditária de Albright. Os dados relativos ao quadro clínico, exames laboratoriais e de imagem, conduta terapêutica, diagnóstico e prognóstico foram obtidos através da consulta e avaliação dos exames do paciente no Centro de Saúde Nova Esperança I, em Bayeux-PB. O caso relatado traz à luz o diagnóstico de uma entidade clínica endocrinológica que tem sua descoberta marcada por manifestações neurológicas e evidencia a importância da sua detecção precoce e abordagem terapêutica adequada, para obtenção de resultados satisfatórios e duradouros no que diz respeito ao controle das crises convulsivas e melhora da qualidade de vida.

PALAVRAS-CHAVE: Hipocalcemia. Pseudo-hipoparatireoidismo. Epilepsia.

Acadêmica de Medicina, Faculdade de Medicina Nova Esperança - FAMENE. Departamento de Medicina. I CEP: 58067-695. João Pessoa, Paraíba, Brasil. *Email do autor correspondente: julya morais@hotmail.com. ORCID ID: 0000-0002-8525-4688; 0000-0002-8747-7391; 0000-0002-3757-9103; 0000-0002-1540-7925

Neurologista. Mestre, Faculdade de Medicina Nova Esperança - FAMENE. Prog. Pós-graduação: Mestrado II Profissional em Saúde da Família. CEP: 58067-695, João Pessoa, Paraíba, Brasil.

ORCID ID: 0000-0002-9412-9096 


\section{INTRODUÇÃO}

O pseudohipoparatireoidismo (PHP) ocorre devido a uma resistência periférica à ação do paratormônio (PTH), cursando com hipocalcemia. Quase todos os casos são do tipo IA, correspondente à Osteodistrofia Hereditária de Albright (OHA), e apresentam manifestações fenotípicas características como baixa estatura, calcificações subcutâneas, braquidactilia, obesidade central e retardo mental. ${ }^{1,2}$

O diagnóstico de pseudohipoparatireoidismo é baseado na condição clínica associada a exames laboratoriais que evidenciam hipocalcemia e hiperfosfatemia, com funções renais normais e concentrações séricas de paratormônio elevadas, enquanto a classificação do tipo de pseudohipoparatireoidismo é feita por investigação genética. ${ }^{13,4}$

A abordagem terapêutica objetiva prevenir complicações agudas e crônicas da doença mantendo adequados níveis séricos

\section{MATERIAL E MÉTODOS}

Trata-se de um estudo retrospectivo, observacional e descritivo com abordagem quantitativa de um paciente portador de Osteodistrofia Hereditária de Albright. Os dados relativos ao quadro clínico, exames laboratoriais e de imagem, conduta terapêutica, de cálcio e, assim, evitando a hipercalciúria e normalizando os níveis de paratormônio e fósforo séricos. O acompanhamento regular é necessário para corrigir medidas terapêuticas e otimizar a evolução do quadro. ${ }^{24,5}$

É importante ressaltar que, apesar do mesmo epônimo, a Osteodistrofia Hereditária de Albright difere da doença de McCune-Albright. Esta última caracterizada por manchas café-com-leite, displasia fibrosa poliostótica, o que resulta em assimetria e fraturas ósseas, alterações endócrinas como puberdade precoce, hipertireoidismo, Síndrome de Cushing, hiperprolactinemia e hiperparatireoidismo. ${ }^{1,2,3}$

O presente estudo tem como objetivo relatar o caso de um paciente de 31 anos, o qual apresentava calcificações nos rins, sistema nervoso central e articulações, além de dosagem baixa do paratormônio, compatível com a Osteodistrofia Hereditária de Albright.

\section{RELATO DE CASO}

TrJ. A. G. M. B., 31 anos, masculino, comparece a consulta no ambulatório de diagnóstico e prognóstico foram obtidos através da consulta e avaliação dos exames do paciente no Centro de Saúde Nova Esperança I, em Bayeux-PB. As informações coletadas durante a pesquisa foram correlacionadas com a literatura médica existente. 
fortes dores na coluna, unhas descalcificadas e câimbras frequentes. Ao exame físico constatou-se fácies sindrômica, baixa estatura, obesidade, prova índex-nariz realizada com dificuldade, bradicinesia, atrofia de membros superiores e inferiores. O paciente apresenta antecedentes pessoais patológicos de epilepsia, diagnosticada desde seus primeiros dias de vida, devido a crises convulsivas tônico-clônico genereralizadas, de duração de 10 segundos, associadas a perda do controle esfincteriano, mordedura de lábio, confusão mental, amnésia retrógrada e sonolência pós-ictal.

O paciente refere ainda a ocorrência de duas a três crises convulsivas ao ano, associadas a presença de calcificações periventriculares e em gânglios da base, evidenciadas em ressonância nuclear magnética (RNM) prévia,

\section{DISCUSSÃO}

Em 1942, Albright descreveu três casos de pacientes que apresentavam alterações analíticas como hipocalcemia, hiperfosfatemia e aumento do paratormônio(PTH)com aparência clínica de hipoparatireoidismo. Como após a administração do hormônio paratireoidiano não ocorria diurese do fosfato nem alterações dos níveis de cálcio e fósforo, esta Síndrome foi nomeada Pseudo-Hipoparatireoidismo (PHP). ${ }^{6}$

Nesses pacientes, encontram-se comumente baixa estatura, face arredondada, pescoço curto, nariz com base larga e dorso achatado, calcificações ou ossificações subcutâneas e braquidactilia, principalmente do quarto e do quinto metacarpos, características essas denominadas de Osteodistrofia Hereditária de Albright. ${ }^{5,7,8,9,10}$. No caso relatado, além das características fenótipicas clássicas como fácies sindromica, baixa estatura e unhas descalcificadas, haviam alterações laboratóriais típicas do Pseudo-Hipoparatireoidismo, as quais estão relacionadas ao quadro de hipocalcemia crônica. Foram solicitadas na ocasião, dosagens de ácido úrico, cálcio iônico, fósforo sérico, fator antinuclear (FAN), fator reumatoide (FR), paratormônio e velocidade de hemossedimentação (VHS). Nessa situação, o paratormônio foi dosado em níveis de $2 \mathrm{pg} /$ $\mathrm{ml}$, com cálcio iônico de $1,07 \mathrm{mmol} / \mathrm{l}$ e fósforo sérico de $6,1 \mathrm{mg} / \mathrm{dL}$, sendo realizado um encaminhamento para o endocrinologista, o qual o diagnosticou com Osteodistrofia Hereditária de Albright e iniciou tratamento com citrato de cálcio e calcitriol. Nesta época, o paciente já apresentava calcificações nos rins, sistema nervoso e articulações, além de um hipoparatireoidismo severo, com riscos de tetania e câimbra.

como o aumento dos níveis séricos de PTH, hipocalcemia, hiperfosfatemia e função renal normal, as quais corroboravam com o diagnóstico.

Décadas depois dos primeiros casos, Albright relata um doente com as mesmas características de Osteodistrofia Hereditária de Albright, mas sem alterações laboratoriais ou evidência de resistência ao PTH, indicando que esse fenótipo característico pode ser herdado separadamente, sem que o doente apresente hipocalcemia ou elevação do paratormônio, nomeando, assim, essa entidade de Pseudopseudo-Hipoparatireoidismo., ${ }^{9,11,12}$

O tipo mais comum de PHP é o IA em que a resistência ao paratormônio é resultante de uma mutação do GNAS1 que codifica a subunidade Gsa da proteína $G$, a qual acopla o receptor do PTH a adenililciclase, necessária para a transdução do sinal aos tecidos alvos endócrinos. Dessa maneira, 
a proteína $G$ é incapaz de ativar a adenilciclase para produzir AMPc que é fundamental para a resposta ao PTH e manutenção da homeostase fosfato-cálcica.9.10,12,13 Considerase as manifestações bioquímicas e os achados clínicos do paciente em estudo, consistentes com um quadro de Osteodistrofia Hereditária de Albright, um pseudohipoparatiroidismo tipo IA.

O Pseudo-Hipoparatireoidismo trata-se de uma desordem hereditária que engloba um grupo heterogêneo de doenças caracterizadas pela resistência dos órgãos-alvo à ação do $\mathrm{PTH}^{8,10,11}$. Pode ser classificado em quatro tipos
(IA, IB, IC e II) de acordo com as manifestações clínicas, os parâmetros bioquímicos e o mecanismo molecular subjacente à resistência hormonal (Tabela 1). ${ }^{14}$

A perda de um alelo funcional que codifica a subunidade Gsa leva a uma deficiência de $50 \%$ das unidades a do heterotrímero Gs, assim a resposta do AMPc à administração do PTH está marcadamente diminuída ou bloqueada. ${ }^{9} 10$ Como a proteína Gs também acopla vários outros receptores à adenilciclase, a resistência parcial a outros hormônios como $\mathrm{TSH}$, gonadotrofinas, $\mathrm{GH}$ e prolactina, pode ser também observada. ${ }^{15}$

TABELA 1: Tipos de Pseudo-Hipoparatireoidismo de acordo com fenótipo, outras resistências hormonais, AMPc e mecanismo.

\begin{tabular}{ccccc}
\hline $\begin{array}{c}\text { Tipo de Peseudo- } \\
\text {-hipoparatireoidismo }\end{array}$ & $\begin{array}{c}\text { Fenótipo Osteodistrofia } \\
\text { hereditária de Albright }\end{array}$ & $\begin{array}{c}\text { Outras resistências } \\
\text { hormonais }\end{array}$ & $\begin{array}{c}\text { AMPc em resposta ao } \\
\text { Pseudo- } \\
\text { hipoparatireoidismo }\end{array}$ & Mecanismo \\
\hline \hline IA & Sim & Sim & Diminuído & Mutação do GNAS1 \\
IC & Sim & Sim & Diminuído & Não elucidado \\
II & Não & Não & Normal elucidado \\
\hline \hline
\end{tabular}

O tipo IA é o mais comum e o IC é o mais raro, o qual apresenta manifestações clinicas e laboratoriais similares ao PHP-IA, distinguindose deste pela ausência de mutação na proteína Gsa. O Pseudopseudo-Hipoparatireoidismo e o Pseudo-Hipoparatireoidismo IA são causados por mutações epigenéticas do gene GNAS1, mapeadas no cromossomo 20q13.3. $\cdot^{10,14}$

O PHP é herdado como um traço autossômico recessivo com modificação relacionada ao sexo, uma vez que quando o defeito no gene GNAS1 tem transmissão materna, a resistência hormonal é expressa em conjunto com o fenótipo da Osteodistrofia Hereditária de Albright. Entretanto, quando o defeito é de transmissão paterna, o fenótipo da Osteodistrofia Hereditária de Albright ocorre isoladamente, pois o alelo materno normal resulta na manutenção a resposta ao PTH., ${ }^{7,12,13,14}$ O fenótipo característico da Osteodistrofia Hereditária de Albright é caracterizadoporfácies emlua cheia, obesidade, baixa estatura, braquidactilia, deformidades articulares (joelho valgo, coxa vara, cubitus valgus) e calcificações subcutâneas, nos rins e no cérebro, sendo a maioria destes, com exceção da braquidactilia, encontrados no paciente apresentado. ${ }^{11}$ Por razões ainda não conhecidas, a maioria dos pacientes que possuem PHP IA tem concentrações normais de cálcio por muitos anos, podendose concluir que a hipocalcemia não é um fator necessário para compor a síndrome, uma vez que normocalcêmicos também podem possuir o fenótipo da Osteodistrofia Hereditária de Albright. 13,7,15 
A Osteodistrofia Hereditária de Albright não é reconhecida normalmente durante os primeiros anos de vida, tornando-se mais evidente aos 4 - 6 anos, quando os níveis de cálcio começam a declinar. No caso em estudo, a única manifestação clínica apresentada durante os primeiros anos, foi o quadro epiléptico. Os sinais e sintomas da hipocalcemia relacionamse com a concentração de cálcio ionizado no soro e, em geral, quanto menores os níveis de cálcio, maior a riqueza e intensidade dos sintomas. ${ }^{9}$

As manifestações clínicas mais características da hipocalcemia aguda incluem câimbras, convulsões, tetania, edema de papila, prolongamento do intervalo QT no eletrocardiograma, insuficiência cardíaca (incomum e reversível diante da reposição de cálcio) e taquicardia., 5,712,13 A hipocalcemia crônica por sua vez, é responsável por alterações cultâneas e dentárias, como a hipoplasia do esmalte dentário nas crianças, catarata, alopécia, retardo mental e calcificação dos gânglios da base, a qual é detectada principalmente pela tomografia computadorizada, e foram descritas no paciente em estudo. ${ }^{4,6,11}$

Embora a tetania e as convulsões sejam as apresentações mais comuns da hipocalcemia em crianças pré-escolares portadoras de PHP IA, algumas delas permanecem assintomáticas até a idade adulta. ${ }^{14,16}$ Além disso, as convulsões também podem aparecer de forma inicial e isolada sem a presença de tetania, como no caso descrito, uma vez que os níveis baixos de cálcio no líquor podem ter efeito convulsivo, mas não tetânico direto. ${ }^{17}$

O diagnóstico da Síndrome é norteado pela anamnese, exame físico e exames complementares. A presença de níveis séricos de PTH aumentados em um indivíduo com hipocalcemia, hiperfosfatemia e função renal normal indicam a possível existência do pseudo- hipoparatireoidismo. ${ }^{1,3,9,14}$ Ao exame físico, os achados clássicos que traduzem irritabilidade neuromuscular são os sinais de Chvostek e Trousseau, porém não foram encontrados no paciente examinado. ${ }^{9}$

A confirmação do diagnóstico clínico pode ser realizada através da existência de uma resposta diminuída do fósforo e AMPc urinários, após a administração de PTH exógeno. O diagnóstico definitivo da Síndrome, bem como a diferenciação dos diversos tipos de PHP são estabelecidos através da mutação da proteína Gsa, contudo não é uma conduta necessária para fixar a abordagem terapêutica nesses pacientes.9,12,13

Além disso, ainda pode-se verificar nos exames de imagem desses pacientes a existência de calcificações subcutâneas, nos gânglios da base e nos rins, como no caso descrito. Embora esses achados radiográficos não sejam específicos, a presença de braquidactilia com calcificações ou ossificações são muito peculiares de Osteodistrofia Hereditária de Albright, cabendo ao radiologista um papel importante no reconhecimento dessa entidade. ${ }^{17}$ No paciente estudado, havia calcificações cerebrais grosseiras vistas à RNM que, somados as alterações eletrolíticas, culminaram nas crises convulsivas e geraram um déficit na capacidade de aprendizado.

Para o diagnóstico diferencial do PHP, deve-se levar em conta as principais causas de hipocalcemia nos indivíduos, tais como o hipoparatireoidismo, a deficiência de vitamina D e o pseudopseudo-hipoparatireoidismo (PPHP). É importante denotar que nem todos os doentes com PHP têm o fenótipo Osteodistrofia Hereditária de Albright entretanto, este está presente em todos os pacientes com PPHP, uma vez que o termo Osteodistrofia Hereditária de Albright é designado para descrever as características físicas, independentemente dos achados bioquímicos (Tabela 2). ${ }^{16}$ 
TABELA 2: Diagnóstico diferencial do pseudo-hipoparatireoidismo

\begin{tabular}{ccccc}
\hline & $\begin{array}{c}\text { Peseudo- } \\
\text {-hipoparatireoidismo }\end{array}$ & Hipoparatireoidismo & $\begin{array}{c}\text { Pseudopseudo- } \\
\text {-hipoparatireoidismo }\end{array}$ & Deficiência vit. D \\
\hline Cálcio & Diminuído & Diminuído & Normal & Diminuído \\
Fósforo & Aumentado & Aumentado & Normal & Diminuído \\
PTH & Aumentado & Diminuído & Normal & Aumentado \\
OHA & $+/-$ & - & Fonte: Adaptado de Vieira \& Vieira16 \\
\hline \hline
\end{tabular}

O objetivo do tratamento nesses pacientes é manter os valores do cálcio ionizado dentro da faixa de normalidade, evitar a hipocalciúria e normalizar os níveis de PTH. Os doentes com hipocalcemia sintomática devem receber suplementação de cálcio e calcitriol, os quais são usados pelo paciente relatado, sendo essa suplementação suficiente também para normalizar o fósforo, fazendo com que os quelantes desse elemento sejam raramente

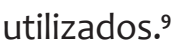

\section{CONCLUSÕES}

O caso relatado e publicações apresentadas trazem à luz uma entidade endocrinológica rara, de prevalência desconhecida devido a escassez de casos, que têm seu diagnóstico marcado primeiramente por manifestações neurológicas e evidencia a importância da sua detecção precoce e abordagem terapêutica adequada, para obtenção de resultados satisfatórios e duradouros no que diz respeito ao controle das crises convulsivas e melhoria da qualidade de vida.

A suspeita da Osteodistrofia hereditária
O seguimento regular dos portadores da Osteodistrofia Hereditária de Albright é essencial para ajustar a terapêutica de acordo com os resultados laboratoriais desses elementos, como também otimizar o metabolismo do fosfato-cálcico. ${ }^{16}$ Se as metas do tratamento forem cumpridas corretamente pode-se reduzir as complicações agudas e crônicas da hipocalcemia, e assim a mortalidade nesses pacientes. ${ }^{16,17}$

de Albright foi baseada nas características fenótipicas clássicas da Síndrome, associadas as alterações neurológicas, laboratoriais e de imagem, prontamente reconhecidas pelo médico neurologista. $O$ diagnóstico definitivo e o tratamento da condição foram estabelecidos pelo endocrinologista referenciado. Devido a magnitude da condição e suas respectivas repercussões clínicas e psicológicas, faz-se necessário também um acompanhamento multidisciplinar nos serviços de neurologia, endocrinologia e psicologia. 


\title{
ALBRIGHT'S HEREDITARY OSTEODYSTROPHY: A CASE REPORT
}

\begin{abstract}
Pseudohypoparathyroidism occurs due to peripheral resistance to parathyroid hormones, resulting in hypocalcemia. Almost every case relates to type IA, which corresponds to Albright's Hereditary Osteodystrophy Syndrome. This study aims to report the case of a 31-year-old patient, who presented calcifications in the kidneys, central nervous system and articulations, as well as low parathyroid hormone levels, compatible with the syndrome mentioned above. This is a retrospective, observational and descriptive study, with a quantitative approach about a patient with Albright's Hereditary Osteodystrophy. The data relating to the clinical manifestations, laboratory and imaging results, therapeutic conduct, diagnosis and prognosis were obtained through consultation and laboratory tests evaluation of the patient at Centro de Saúde Nova Esperança I, in Bayeux-PB. The reported case brings to light the diagnosis of an endocrine disorder, which was primarily discovered through neurological manifestations, reinforcing the importance of an early detection and adequate therapeutic approach in order to reach satisfying and lasting results regarding the control of seizure episodes and improve quality of life.
\end{abstract}

KEYWORDS: Hypocalcemia. Pseudohypoparathyroidism. Epilepsy.

\section{REFERÊNCIAS}

1. Tafaj, O, Juppner, H. J. Pseudohypoparathyroidism: one gene, several syndromes. Journal of Endocrinological Investigation. 2017; 40(4): 34753.

2. Trejo MC, Román GA, Ruíz S, Tobón C, Castaño $\mathrm{P}$, Arango $\mathrm{C}$, et al . Late diagnosis of pseudohypoparathyroidism in adulthood: Case series. Rev Fac Med. 2018; 66(4): 643-49.

3. Sethuraman G, Malhotra AK, Khaitan BK, Kumar R, Sharma VK, Kabra M, et al. Osteoma cutis in pseudohypoparathyroidism. Clin Exp Dermatol. 2006; 31(2): 225-27.

4. Friederich SA, Rodrigues J, Silva BB. Albright hereditary osteodystrophy: dental management case report. Rev Gaúch Odontol. 2018; 66(1): 106-10.

5. Mamoei S, Cortnum S. Raised intracranial pressure as a result of pansynostosis in a chidl with Albright's hereditary osteodystrophy. Childs Nerv Syst. 2017; 33(5): 865-68.

6. Albright $\mathrm{F}$, Burneti $\mathrm{CH}$, Smith $\mathrm{PH}$, Parson W. Pseudohypoparathyroidism: An example of "Seabright-Bantan" Syndrome. Endocrinology. 1942; 30(2): 922-32.

7. Martos-Moreno GA, Lecumberri B, de Nanclares GP. Implicaciones en pediatría del primer consenso internacional para el diagnóstico y asistencia a pacientes con pseudohipoparatiroidismo 
y enfermedades relacionadas. Anales de Pediatría. 2019; 90(2): 125-37.

8. Taiti N, Riu MR, Segui NE, Burguesa FR. Pseudopseudohipoparatiroidismo, a propósito de un caso. Rev Esp Endocrinol Pediatr. 2015; 6(1): 5154.

9. Campos R, Vilar L, Moura E, Carvalho MA, Bandeira F. Doenças Osteometabólicas. In: Vilar L. Endocrinologia Clínica. 6. ed. Rio de Janeiro: Guanabara Koogan; 2016. p.813-15.

10. Miyakawa Y, Takasawa K, Matsubara Y, Ihara K, Ohtsu Y, Kamasaki H, et al. Language delay and developmental catch-up would be a clinical feature of pseudohypoparathyroidism type $1 \mathrm{~A}$ during childhood. Endocrine Journal. 2019; 66(3): 215-21.

11. Underbjerg L, Sikjaer T, Mosekilde L, Rejnmark, L. Pseudohypoparathyroidism: Epidemiology, mortality, and risk of complications. Clin Endocrinol. 2016; 84(6): 904-11.

12. Reis MTA. Avaliação fenotípica e de defeitos moleculares no GNAS em pacientes com pseu- do-hipoparatireoidismo [tese doutorado]. São Paulo: Universidade de São Paulo; 2014.

13. Elli FM, de Sanctis L, Ceoloni B, Barbieri AN, Bordogna P, Beck-Peccoz P, et al. Pseudohypoparathyroidism type la and pseudo-pseudohypoparathyroidism: the growing spectrum of GNAS inactivating mutations. Human Mutation. 2013; 34(3): 411-16.

14. Burgert T, Markowitz M. Understanding and Recognizing Pseudohypoparathyroidism. Pediatr Rev. 2005; 26(8): 308 -09.

15. Peña C, Pinochet C, Florenzano P, Mendoza C, Garfias C, Aracena M et al. Pseudohipoparatiroidismo de presentación tardía: reporte de dos casos. Rev Med Chile. 2018; 146(1): 116-21.

16. Vieira H, Vieira P. Um caso clínico de pseudohipoparatiroidismo. Nascer e Crescer. 2011; 20(4): 266-69.

17. Rodrigues MC, Correia R., Carvalho A, Portugal I, Rebelo J, Viamonte B. Osteodistrofia hereditária de Albright: alterações radiográficas. Acta Radiológica Portuguesa. 2017; 29(3), 45-48. 